This item was submitted to Loughborough's Research Repository by the author.

Items in Figshare are protected by copyright, with all rights reserved, unless otherwise indicated.

\title{
What governs successful performance of a complex whole body movement: The Kovacs release-regrasp on horizontal bar?
}

PLEASE CITE THE PUBLISHED VERSION

http://dx.doi.org/10.1016/j.jbiomech.2016.11.048

\section{PUBLISHER}

(C) Elsevier

\section{VERSION}

AM (Accepted Manuscript)

\section{PUBLISHER STATEMENT}

This work is made available according to the conditions of the Creative Commons Attribution-NonCommercialNoDerivatives 4.0 International (CC BY-NC-ND 4.0) licence. Full details of this licence are available at: https://creativecommons.org/licenses/by-nc-nd/4.0/

\section{LICENCE}

CC BY-NC-ND 4.0

\section{REPOSITORY RECORD}

Hiley, Michael, and Fred Yeadon. 2019. "What Governs Successful Performance of a Complex Whole Body Movement: The Kovacs Release-regrasp on Horizontal Bar?”. figshare. https://hdl.handle.net/2134/23900. 


\title{
What governs successful performance of a complex whole body movement: the Kovacs release-regrasp on horizontal bar?
}

\author{
Michael J. Hiley ${ }^{1}$ and Maurice R. Yeadon ${ }^{1}$ \\ ${ }^{1}$ School of Sport, Exercise \& Health Sciences, Loughborough University, Loughborough, UK
}

\begin{abstract}
The Kovacs is a release and regrasp skill performed on the horizontal bar in men's artistic gymnastics. It is a popular skill in elite competitive gymnastics with over $40 \%$ of male gymnasts performing a variation of the Kovacs at the London 2012 Olympics. In the qualifying competition $84 \%$ of Kovacs were successfully regrasped, with the remaining $16 \%$ resulting in a fall. The aim of the present study was to determine why some gymnasts are more successful than others at regrasping the bar, with a secondary aim to determine how a less successful gymnast could alter his technique in order to become more successful. Nine performances of the Kovacs by each of two gymnasts, one $100 \%$ successful and one $11 \%$ successful, were analysed to determine differences in release and regrasp parameters. The technique of the less successful gymnast was optimised using a computer simulation model to increase the percentage of catches (success rate). The successful gymnast had larger and more consistent release windows and a radial velocity towards the bar at regrasp. The less successful gymnast had higher horizontal velocity at release and a mean radial velocity away from the bar at regrasp. Optimising his simulated technique increased the rate of success from $11 \%$ to $93 \%$. The actions prior to release were performed earlier than in the recorded performances leading to a more vertical path of the mass centre at release and a radial velocity towards the bar at regrasp.
\end{abstract}

\section{INTRODUCTION}

The Kovacs is a release and regrasp skill performed on the horizontal bar in men's artistic gymnastics. From a backward giant circle (longswing) the gymnast releases the bar and rotates through approximately one and a quarter somersaults, regrasps the bar and then continues to swing in the backwards direction (Figure 1). The Kovacs is a popular skill in elite competitive gymnastics. At the London 2012 Olympic Games over $40 \%$ of male gymnasts performed a variation (body shape and twists) of the Kovacs in competition 1 (qualifying). Although popular, it is not uncommon for gymnasts to miss the regrasp, resulting in a fall and a large points deduction from the judges (FIG, 2013). In the qualifying competition at the London Olympics $84 \%$ of Kovacs performed were successfully regrasped, with the remaining $16 \%$ resulting in a fall. Since the gymnast is a projectile once the bar has been released, the main reason for missing the regrasp may be due to having inappropriate amounts of linear and angular momenta at release (Holvoet et al., 2002; Hiley et al., 2007; Hiley and Yeadon, 2012a). Holvoet et al. (2002) used a simulation model to demonstrate that an unsuccessful Tkatchev (a counter rotation release and regrasp skill, Figure 1) could have been caught if the gymnast had released the bar earlier than in the recorded performance. In contrast, Hiley et al. (2007) found that for a different gymnast performing unsuccessful Tkatchevs, an earlier release would not have resulted in the gymnast being able to regrasp the bar. This gymnast would have had to change his giant circling technique in order to be successful. 

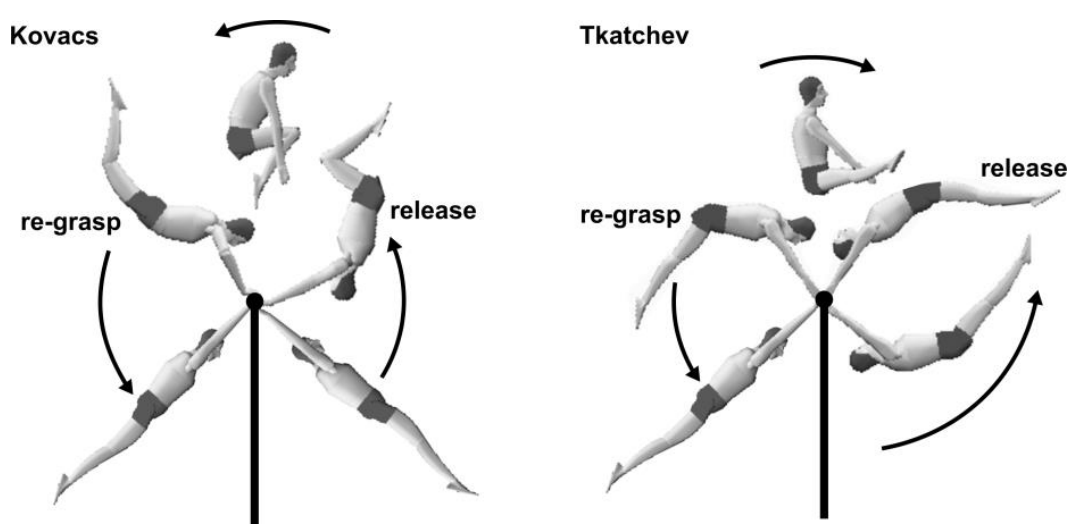

Figure 1. The Kovacs and the Tkatchev release and regrasp on horizontal bar.

In addition to having appropriate release conditions, the size of the release window (Hiley \& Yeadon, 2003a) has been shown to have an impact on the success of a release skill (Hiley and Yeadon, 2016). The release window has been defined as the timing window within which the gymnast can release the bar and successfully complete the following skill. For release and regrasp skills there will also be a regrasp window, which can be defined as the time period during which a successful regrasp of the bar can be made. Understanding such constraints may provide insight into why some gymnasts are more successful at consistently regrasping the bar.

Hiley and Yeadon (2012a) demonstrated that the success rate (percentage of successful regrasps) for the Tkatchev was predominantly affected by the gymnast's technique. The strength and coordination precision of the gymnast were also found to have substantial effects on success rate. The coordination precision was subsequently used to represent the level of noise within the motor system that the gymnast must cope with in an upstart (Hiley and Yeadon, 2014). An athlete's technique must be robust to the level of noise within the motor system, so that a successful performance can be achieved even when aspects of the technique are not performed exactly as planned (van Beers et al., 2004; Wilson et al., 2007; Cohen and Sternad, 2009; Hiley and Yeadon, 2016). Thus unsuccessful performance is not always due to mistiming the release.

The aim of the present study is to determine why some gymnasts are more successful with the Kovacs than others. It is hypothesised that a successful gymnast will have larger and more consistent release windows, based on linear and angular momenta at release and the subsequent regrasp window. It is also hypothesised that a successful gymnast will have a more consistent technique than a less successful gymnast. A secondary aim will be to use a computer simulation model to determine how a less successful gymnast could alter his technique in order to become more successful at the Kovacs release and regrasp.

\section{METHOD \\ Data collection}

Two male gymnasts (age 21/22 years, mass 70/68 kg, height 1.62/1.68 m) who competed internationally with a Kovacs in their competitive routine gave informed consent to participate in the study which was approved by the university ethics committee. The gymnasts each performed nine trials of the Kovacs which were captured using 15 Vicon MX13 cameras operating at $300 \mathrm{~Hz}$. Gymnast 1 successfully regrasped nine out of nine trials, whereas Gymnast 2, successfully regrasped once and touched the bar without regrasping on four of the remaining 
eight trials. Spherical reflective markers, $25 \mathrm{~mm}$ in diameter, were attached to the lateral side of the wrist, elbow, shoulder, hip, knee and ankle joint centres and toes on the left side of the body. Additional markers were attached to each side of the gymnast's head (above the ear) and to the centre of the bar. Prior to data collection a volume centred on the bar spanning $2 \mathrm{~m} \times 5 \mathrm{~m} \times 5 \mathrm{~m}$ was wand calibrated using the motion analysis system. Three-dimensional marker coordinates were reconstructed and joint centre locations were determined using measured offsets (Hiley and Yeadon, 2016). The data in each trial for the last $13 / 4$ giant circles and the aerial phase were analysed.

\section{Data Analysis}

The gymnast mass centre location was calculated from the reconstructed joint centres and gymnast-specific inertia data obtained from anthropometric measures and the geometric inertia model of Yeadon (1990a). The point of release and regrasp (where appropriate) were determined using the displacement of the wrist from the centre of the bar (Kerwin et al., 1993). The horizontal and vertical mass centre velocity at release were determined from the mass centre trajectory in flight using equations of motion under constant acceleration. The method of Yeadon (1990b) was used to determine the normalised angular momentum (in straight somersaults per flight time) at the instant of release for each trial.

The individual level of kinematic variability in the gymnasts' giant circling technique was determined from the joint angle time histories at the shoulder and hip (Hiley et al., 2013) which were normalised to the average duration of the 9 trials for each gymnast (bar contact phase). The average durations for Gymnast 1 and Gymnast 2 were $2.30 \mathrm{~s}$ ( \pm 0.01 , standard deviation) and $2.20 \mathrm{~s}( \pm 0.02)$, respectively. Temporal and spatial kinematic variability was defined as the standard deviations at 12 turning points between flexion and extension at the shoulder and hip (i.e. six turning points at the shoulder and six at the hip) during the giant circles before release (Figure 2 and Table 1). The angle, time and rotation angle (the angle between the upward vertical and the line from the neutral bar position to the model mass centre) were recorded at each of the 12 extrema and the mean and standard deviation were calculated for each gymnast (Hiley et al., 2013).

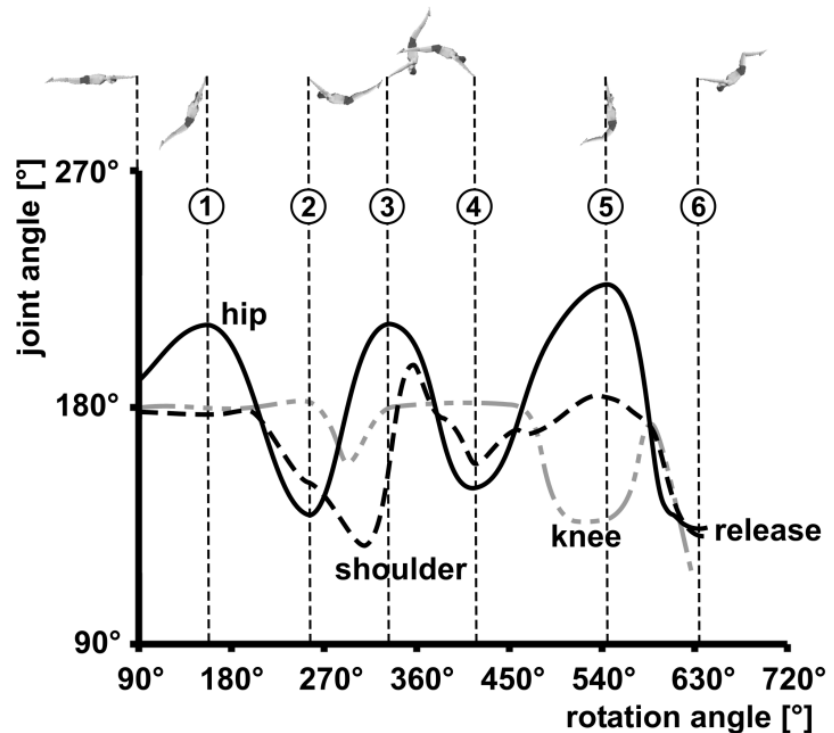

Figure 2. Joint angle time histories of the hip and shoulder with the maximum and minimum flexion/extension angles indicated along with a graphical representation of the gymnast. 
Table 1. Standard deviations in the temporal and spatial dimensions at the extrema of the hip and shoulder time histories along with the rotation angle for the nine Kovacs trials of each gymnast

\begin{tabular}{|c|c|c|c|c|c|c|c|c|c|c|c|c|}
\hline \multirow{2}{*}{$\begin{array}{l}\text { Standard } \\
\text { deviation }\end{array}$} & $\begin{array}{l}\text { Hip } \\
\text { Max }\end{array}$ & $\begin{array}{l}\text { Hip } \\
\text { Min }\end{array}$ & $\begin{array}{l}\text { Hip } \\
\text { Max }\end{array}$ & $\begin{array}{l}\text { Hip } \\
\text { Min }\end{array}$ & $\begin{array}{l}\text { Hip } \\
\text { Max }\end{array}$ & $\begin{array}{l}\text { Hip } \\
\text { Min }\end{array}$ & $\begin{array}{l}\text { Shld } \\
\text { Max }\end{array}$ & $\begin{array}{l}\text { Shld } \\
\text { Min }\end{array}$ & $\begin{array}{l}\text { Shld } \\
\text { Max }\end{array}$ & $\begin{array}{l}\text { Shld } \\
\text { Min }\end{array}$ & $\begin{array}{l}\text { Shld } \\
\text { Max }\end{array}$ & $\begin{array}{l}\text { Shld } \\
\text { Min }\end{array}$ \\
\hline & (1) & (2) & (3) & (4) & (5) & (6) & (1) & (2) & (3) & (4) & (5) & (6) \\
\hline \multicolumn{13}{|l|}{ Gymnast 1} \\
\hline Temporal [ms] & 3 & 7 & 14 & 9 & 5 & 7 & 3 & 6 & 9 & 21 & 4 & 2 \\
\hline Spatial $\left[^{\circ}\right]$ & 0.8 & 0.6 & 1.3 & 3.4 & 1.3 & 1.3 & 0.5 & 6.9 & 0.7 & 2.1 & 1.0 & 0.9 \\
\hline Rot. Ang. $\left[{ }^{\circ}\right]$ & 0.5 & 1.5 & 2.4 & 2.3 & 1.4 & 2.4 & 1.2 & 1.3 & 1.3 & 4.7 & 2.1 & 1.0 \\
\hline \multicolumn{13}{|l|}{ Gymnast 2} \\
\hline Temporal [ms] & 7 & 8 & 8 & 14 & 4 & 23 & 7 & 8 & 6 & 7 & 5 & 12 \\
\hline Spatial $\left[^{\circ}\right]$ & 1.4 & 1.5 & 1.1 & 1.6 & 0.8 & 2.4 & 0.4 & 0.8 & 0.5 & 1.2 & 0.7 & 0.5 \\
\hline Rot. Ang. $\left[^{\circ}\right]$ & 1.6 & 1.5 & 1.4 & 3.4 & 1.8 & 7.5 & 1.7 & 1.4 & 0.8 & 1.8 & 1.6 & 3.6 \\
\hline
\end{tabular}

\section{Simulation model}

In order to determine the release window for each trial a planar four-segment angle-driven model of a gymnast (comprising arm, torso, thigh and lower leg segment) and bar was used (Hiley and Yeadon, 2003a). The bar and the gymnast's shoulder structure were modelled using damped linear springs (Figure 3). The spring at the shoulder represented the increase in length of the gymnast between the wrist and the hip. In addition the torso segment was allowed to lengthen as the shoulder elevation angle increased (Begon et al., 2008).

Model parameters included gymnast-specific segmental inertia data, stiffness and damping coefficients of the springs, and the torso lengthening parameter. The initial conditions comprised the initial displacement and velocity of the bar, and the angular velocity and orientation of the arm. Input to the model comprised the joint angle time histories of the shoulder, hip and knee in the form of quintic splines (Wood and Jennings, 1979). Output from the model included the time histories of the bar displacements, the rotation angle, the linear and angular momenta of the model about its mass centre, and joint torque time histories. The equations of motion were derived using Newton's Second Law and by taking moments about the neutral (unloaded) bar position and the segment mass centres (Hiley and Yeadon, 2003a).

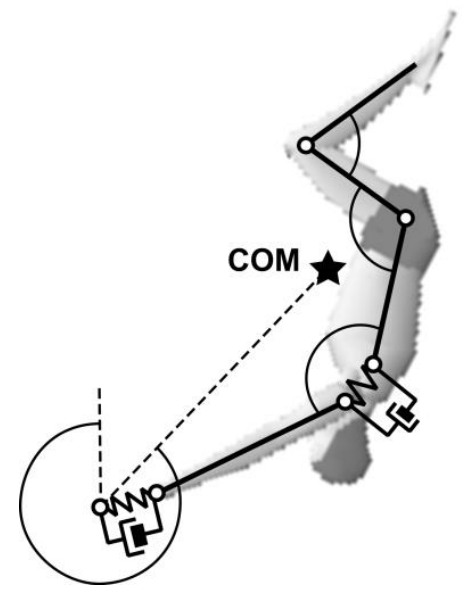

Figure 3. Simulation model of the gymnast and high bar with the rotation angle and joint angle definitions. 


\section{Matching Simulations and Release Window}

For each of the nine Kovacs of each gymnast the release window was determined from a matching simulation. A simulation model was used since the release window starts before and ends after the gymnast has released the bar. The matching procedure was used to produce a common set of model parameters for the nine trials (Hiley and Yeadon, 2016). All matching simulations for each gymnast used the same set of model parameter values determined from concurrent matching optimisations, but with the initial conditions and joint angle time histories from the individual recorded performances. The match was based on root mean squared (rms) differences between the recorded and simulated rotation angle and bar displacements along with differences between recorded and simulated linear and angular momenta at release. The model was able to match, on average over the 18 trials, the rotation angle to within $1^{\circ}$ and the release momenta to within $1 \%$ of the recorded values (Table 2).

Table 2. Release variables and windows determined from the nine Kovacs of each gymnast

\begin{tabular}{|c|c|c|c|c|c|c|c|}
\hline & Trial & $\begin{array}{c}\text { Angular } \\
\text { momentum* } \\
\text { [ss] }\end{array}$ & $\begin{array}{c}\text { Horizontal } \\
\text { velocity } \\
{\left[\mathrm{ms}^{-1}\right]}\end{array}$ & $\begin{array}{l}\text { Vertical } \\
\text { velocity } \\
{\left[\mathrm{ms}^{-1}\right]}\end{array}$ & $\begin{array}{c}\text { Rotation } \\
\text { angle } \\
\left.{ }^{\circ}\right]\end{array}$ & $\begin{array}{c}\text { Release } \\
\text { window } \\
{[\mathrm{ms}]}\end{array}$ & $\begin{array}{c}\text { Release } \\
\text { window } \\
{\left[{ }^{\circ}\right]}\end{array}$ \\
\hline \multirow[t]{11}{*}{ Gymnast 1} & 1 & 0.96 & 1.36 & 4.38 & 660.0 & 135 & 49 \\
\hline & 2 & 0.95 & 1.56 & 4.37 & 665.1 & 125 & 45 \\
\hline & 3 & 0.96 & 1.39 & 4.32 & 664.6 & 137 & 49 \\
\hline & 4 & 1.03 & 1.26 & 4.56 & 653.8 & 119 & 41 \\
\hline & 5 & 0.97 & 1.67 & 4.38 & 663.0 & 117 & 43 \\
\hline & 6 & 0.95 & 1.48 & 4.33 & 663.1 & 145 & 52 \\
\hline & 7 & 0.94 & 1.39 & 4.37 & 663.5 & 138 & 50 \\
\hline & 8 & 0.96 & 1.46 & 4.44 & 661.6 & 156 & 55 \\
\hline & 9 & 0.95 & 1.45 & 4.35 & 663.2 & 156 & 55 \\
\hline & Mean & 0.96 & 1.44 & 4.63 & 662.0 & 136 & 49 \\
\hline & Stdev & 0.02 & 0.11 & 0.08 & 0.10 & 14 & 5 \\
\hline \multirow[t]{11}{*}{ Gymnast 2} & 1 & 1.01 & 1.82 & 4.82 & 640.9 & 99 & 34 \\
\hline & 2 & 0.97 & 2.03 & 4.69 & 643.4 & 50 & 17 \\
\hline & 3 & 0.97 & 2.00 & 4.67 & 645.4 & 56 & 19 \\
\hline & 4 & 0.95 & 1.92 & 4.76 & 640.5 & 66 & 22 \\
\hline & 5 & 1.01 & 1.84 & 4.87 & 637.5 & 72 & 25 \\
\hline & 6 & 1.04 & 1.83 & 4.80 & 641.9 & 84 & 29 \\
\hline & 7 & 0.95 & 1.71 & 5.04 & 638.7 & 60 & 21 \\
\hline & 8 & 1.02 & 1.93 & 4.69 & 644.5 & 67 & 23 \\
\hline & 9 & 0.98 & 2.01 & 4.82 & 637.8 & 43 & 14 \\
\hline & Mean & 0.99 & 1.90 & 4.80 & 641.2 & 66 & 22 \\
\hline & Stdev & 0.03 & 0.11 & 0.11 & 2.9 & 17 & 6 \\
\hline
\end{tabular}

* Normalised to straight somersaults per flight time

From the matching simulation of each trial the start and end of the release window was defined such that the normalised angular momentum, horizontal and vertical velocity of the mass centre lay within three standard deviations (SD) of the mean values obtained from the nine trials (Table 2). In addition, for every time step of the matching simulation the flight parabola was calculated to ensure that the gymnasts mass centre was in an acceptable position to regrasp the bar (catching window). For Gymnast 1, the catching window was defined as the period of time the mass centre 
lay within $\pm 3 \mathrm{SD}$ of the mean recorded catching location from the nine successful trials (Figure 4). An additional constraint was imposed on the catching window so that the gymnast's mass centre location did not exceed the recorded maximum distance from the bar at regrasp (Figure 4). For Gymnast 2, since the mass centre location at regrasp for the one successful trial lay within the range obtained for Gymnast 1 (Figure 4), the same catching window criteria were used.

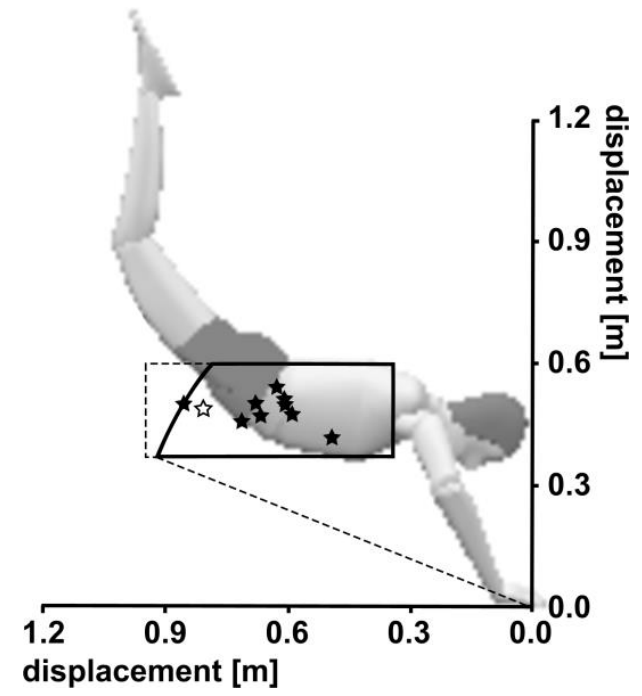

Figure 4. Definition of the catching window (solid line), with centre of mass location at regrasp for the gymnast 1 (black stars) and the one successful trial of gymnast 2 (clear star).

\section{Statistical Analysis}

Linear velocity, angular momentum and rotation angle at release along with the size of release window were compared for the successful and less successful gymnast. The Shapiro Wilk test was used to determine that not all of the data sets were normally distributed. In order to determine if there were differences in performance between the two gymnasts, Mann Whitney $U$ tests were carried out. Effect sizes were calculated as the difference of means divided by the pooled variance (Cohen, 1988). Similarly, to establish whether Gymnast 1 had a more consistent technique than Gymnast 2, Mann Whitney U tests were conducted for the kinematic variability data in Table 1 . All statistics were carried out using SPSS.

\section{Optimisation}

The technique of Gymnast 2 was optimised to increase the likelihood of successfully regrasping the bar. The optimisation score was based on "maximising success" (Hiley and Yeadon, 2014), where success was defined as a solution that produced joint angle time histories that did not exceed the strength characteristics of the gymnast, produced a release window at least as large as the mean of Gymnast 2 (66 ms, Table 2) and was able to cope with the level of noise from the motor system (Hiley and Yeadon, 2014). Analysis of both gymnasts showed that Gymnast 1 had a mean radial velocity towards the bar of $1.34 \mathrm{~ms}^{-1}$ when regrasping whereas Gymnast 2 had a mean radial velocity of $0.20 \mathrm{~ms}^{-1}$ away from the bar (Figure 5). Therefore, an additional constraint was placed on the release window, namely that the model was required to have a radial velocity of at least $0.81 \mathrm{~ms}^{-1}$ towards the bar (the radial velocity at regrasp from Gymnast 2's successful trial) throughout the catching window. 

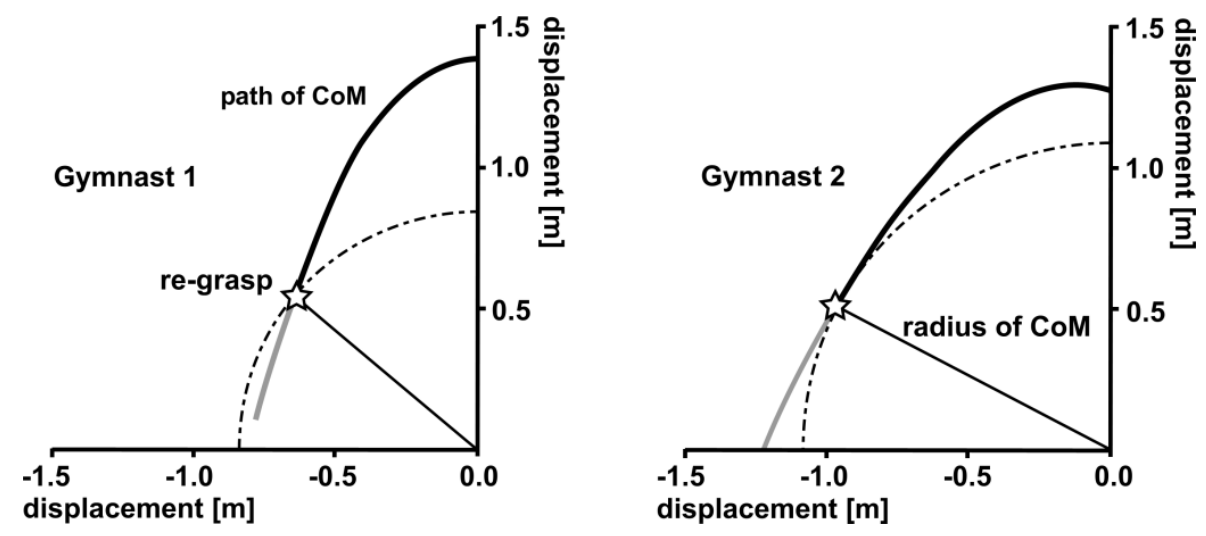

Figure 5. The radial velocity of the mass centre at regrasp was determined as the rate of change of the distance from the mass centre to the bar. Gymnast 1 had a mean radial velocity towards the bar, Gymnast 2 had a mean radial velocity away from the bar.

All simulations started from a rotation angle of 90 (Figure 2), with initial angular velocity from the average trial of Gymnast 2, and ended once the rotation angle passed $690^{\circ}$. The optimisation procedure used a parallelised genetic algorithm run on a high performance computer with 60 processors (Carroll, 2001; van Soest and Cassius, 2003). The recommended optimisation algorithm tuning was used except for the selection of single point crossovers (Carroll, 2001). As in Hiley and Yeadon (2016), single point crossovers were chosen so that sections of joint angle time histories could be passed on to the offspring rather than just single parameter values. The optimisation algorithm manipulated 12 time and 12 angle parameters (24 in total) which defined the joint angle time histories at the shoulder, hip and knee. These were the maximum and minimum points in the mean joint angle time histories from the 9 recorded performances which were used in the manipulation of the joint angle time histories (Figure 2). The average time history of each joint angle over the nine trials was transformed between consecutive maximum/minimum in both the time and angle dimensions (Hiley and Yeadon, 2013). The bounds on the joint angle and timing parameter changes were chosen to perform a local optimisation: $\pm 10^{\circ}$ and $\pm 100 \mathrm{~ms}$ around the values obtained from the average trial. Since Gymnast 2 was capable of regrasping the bar, the optimisation was used to determine what small changes would be required to make him sufficiently successful (i.e. greater than the $84 \%$ success rate from the Olympics in 2012). All joint angle histories were constrained using joint torque limits determined from isovelocity dynamometer measurements, by fitting a function which expressed maximum voluntary torque in terms of joint angle and angular velocity (Forrester et al., 2011). The functions were scaled using joint torques from the matched simulations (Hiley and Yeadon, 2016; King, et al., 2009) so that the torques used in simulations lay within those of the recorded performances.

In order to incorporate noise from the motor system, the parameters defining the joint angle time histories of the shoulder, hip and knee were randomly perturbed, in both the time and angle dimension, to the level of the mean kinematic variability measured in the gymnast performances, standard deviations of $1.5^{\circ}$ and $8 \mathrm{~ms}$ (Table 1). Perturbations were added to the joint angle time history parameters using a random number generator with a normal distribution. For each set of joint angle parameters produced by the optimisation algorithm, 500 randomly perturbed simulations were performed. Simulations were given a score of 1 for a successful 
simulation and 0 for an unsuccessful simulation, with the percentage of successful simulations out of 500 returned as the score for that set of parameters.

\section{RESULTS}

Gymnast 1 was more successful at regrasping the bar (100\% successful, nine out of nine trials) than Gymnast $2(11 \%$ successful). Significant $(p<0.001)$ differences were found between the two gymnasts for all release variables (all effect sizes > 1.7), with the exception of the normalised angular momentum $(p=0.066)$. Gymnast 2 (less successful) had significantly higher horizontal and vertical velocity at release (Table 2). Gymnast 2 also released the bar, on average, significantly earlier in the giant circle and had smaller release windows than Gymnast 1 (Table 2). As hypothesised, the successful gymnast (Gymnast 1) had both larger and more consistent release windows compared to Gymnast 2 (Table 2). It had been hypothesised that the successful gymnast would have a more consistent technique; however Mann Whitney $U$ tests performed on the kinematic variability data (Table 1) found no significant differences between the two gymnasts $(p>0.27)$.

The results of the local optimisation to increase the success rate of Gymnast 2, found a solution that would have increased the gymnast's success rate from $11 \%$ to $93 \%$. The solution resulted in a more vertical path of the mass centre and a larger release window (Figure 6a). The optimal solution used an earlier opening of the hip and shoulder angles in the final giant circle, which resulted in an earlier initiation of the closing of both angles (Figure 6b). A later extension and then flexion of the knees prior to release was also observed.

(a)

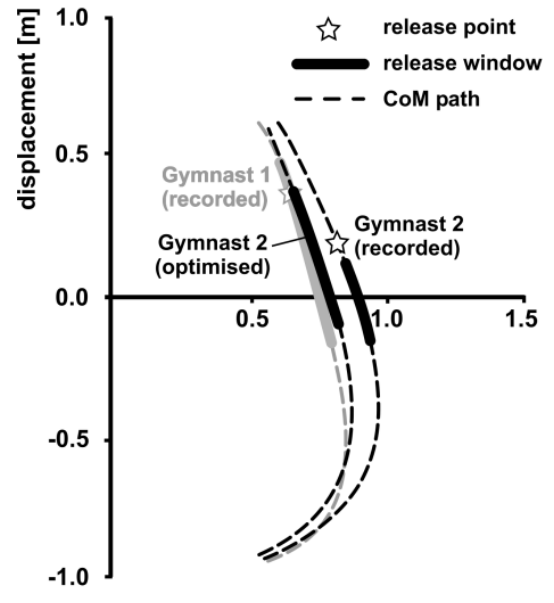

(b)

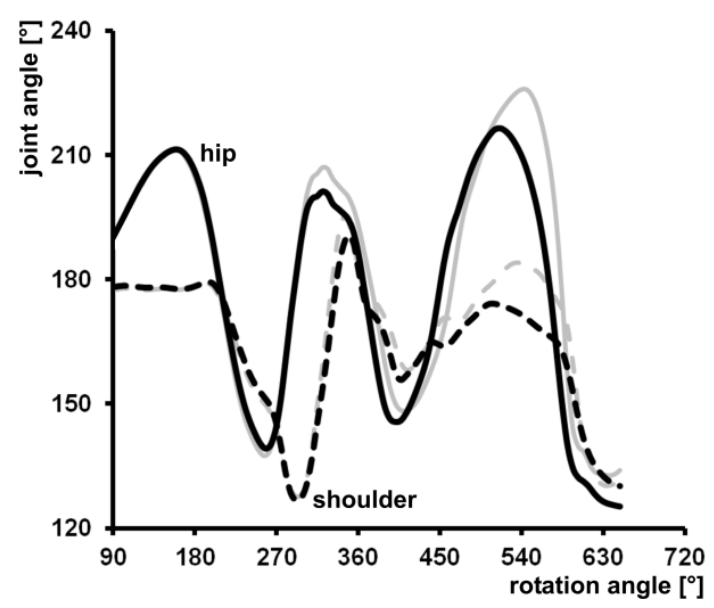

Figure 6. (a) Recorded path of the centre of mass through release for Gymnast 1 (grey line) and Gymnast 2 (black) along with the path of the centre of mass for the optimised solution with the release windows shown (thicker portions of the lines). (b) Joint angle time histories for the hip (solid lines) and shoulder (dashed lines) for the average technique of Gymnast 2 (grey lines) and optimal solution (black lines).

\section{DISCUSSION}

Although only two gymnasts participated, the full range of success was spanned in that one gymnast was maximally successful (100\%) while the other was minimally successful (11\%). Despite significant differences in most of the release variables, each gymnast was very consistent in terms of the linear and angular momentum at release (Table 2). Previous research has reported mean release angles for the Kovacs of $640^{\circ} \pm 9^{\circ}$ (Irwin et al., 2016) and $660^{\circ} \pm 7^{\circ}$ (Brüggemann et al., 1994) with 
mean horizontal and vertical velocities at release ranging from $1.60 \mathrm{~ms}^{-1}$ to $1.64 \mathrm{~ms}^{-1}$ and $4.21 \mathrm{~ms}^{-1}$ to $4.78 \mathrm{~ms}^{-1}$, respectively (Brüggemann et al., 1994; Arampatzis and Brüggemann, 1999; Irwin et al., 2016). These data were reported from the 1994 World Championships and the 1992 and 2000 Olympic Games. It is noted that Gymnast 1 released the bar at the higher end of the reported release angles (Table 2 ) but with lower horizontal velocity $\left(1.44 \mathrm{~ms}^{-1}\right)$. This confirms that Gymnast 1 had a more vertical path of the mass centre (Figure 6a), which in turn led to larger release windows compared to Gymnast 2. Although Gymnast 2 released the bar at a similar rotation angle and with a similar vertical velocity, compared to the previous research (Table 2), his horizontal velocity was considerably higher $\left(1.90 \mathrm{~ms}^{-1}\right)$ than all other gymnasts. It might be expected that Gymnast 2's success rate would be improved by having a more vertical path of the mass centre (i.e. lower horizontal velocity at release).

The analysis of the release windows showed that Gymnast 1 had significantly larger windows than Gymnast 2 (Table 2). However, Gymnast 2 did have reasonably large release windows (Table 2) compared to previous studies on release skills (Hiley and Yeadon, 2016). The release windows of Gymnast 2 were comparable to those found for a gymnast performing repeated trials of the double layout somersault dismount from high bar, $57 \mathrm{~ms} \pm 12 \mathrm{~ms}$ (Hiley and Yeadon, 2016), where the release window was defined using similar constraints, \pm 3 SD of the recorded release parameters. The successful trial of Gymnast 2 released within the release window, as did the four trials that touched the bar. The remaining four trials released outside the release window. This helps explain why Gymnast 2 was able to touch the bar on five of the trials. If Gymnast 2 had released earlier on the remaining four trials (within the release window) he would have been able to touch the bar. This raises the question, if Gymnast 2 had sufficiently large release windows and was in range to regrasp the bar, why did he not catch the bar on more than one of the nine attempts? In order to understand why, further analysis of release parameters was undertaken.

Analysis of Gymnast 1 showed that on average release occurred $9^{\circ}$ before the end of the release window. A large portion of the release window was therefore not being used, as the gymnast was choosing when in the window to release. Therefore, simply maximising the release window of Gymnast 2 may not be beneficial. It appears as though the horizontal velocity at release is the key factor for a successful regrasp. When the gymnast opens from the tuck position and views the bar, an evaluation of when to regrasp will be made. With higher than optimal horizontal velocity the gymnast will not be in the correct location and will be travelling faster than expected. Having a large horizontal velocity whilst regrasping the bar may also not be desirable, leading to potentially high loading and the possibility of losing grip of the bar (referred to as "pinging"). The two consequences of having a high horizontal mass centre velocity may help explain the lack of success at regrasping the bar. Inspection of the mass centre radial velocity of the two gymnasts at regrasp (closest wrist location to the bar for the unsuccessful trials), showed that Gymnast 1 had a mean radial velocity towards the bar of $1.3 \mathrm{~ms}^{-1}$ (Figure 5), whereas Gymnast 2 had a mean radial velocity away from the bar of $0.2 \mathrm{~ms}^{-1}$. The radial velocity was calculated as the rate of change of the distance from the neutral bar location to the mass centre (Figure 5). Gymnast 2 had a radial velocity of $0.8 \mathrm{~ms}^{-1}$ towards the bar at regrasp on his one successful trial. With a radial velocity towards the bar the gymnast can regrasp and push away from the bar. With a radial velocity away from 
the bar, the gymnast must overcome this velocity and the associated reaction force to avoid "pinging" from the bar.

Gymnast 2's technique was as consistent as Gymnast 1's, suggesting that some aspect was being performed incorrectly. Gymnast 2's technique was optimised to increase the success rate. In addition to being in the correct location at regrasp the model was encouraged to have a radial velocity towards the bar. Using a local optimisation (limited bounds) the success rate was increased from $11 \%$ to $93 \%$. The optimisation was termed a local optimisation since only relatively small changes in technique were permitted. The most notable change in technique was the rotation angle at peak hip hyper-extension and the subsequent hip flexion. The hyperextension occurred earlier in the circle and was less pronounced, and the hip flexion which was over a similar angle range, was performed over a larger rotation angle (Figure 6b). A similar pattern was also seen at the shoulder (Figure 6b). The earlier initiation of the closing of the shoulder and hip angles in the circle resulted in a slightly more vertical path of the mass centre (Figure 6a), leading to a radial velocity towards the bar at regrasp. The result demonstrates how important the technique through the lower part of the circle is. As with other horizontal bar release skills (Gervais and Tally, 1993; Hiley and Yeadon, 2003b; Hiley and Yeadon, 2012a, 2012b; Irwin et al., 2016), the timing of closing joint angles has an effect on the loading of the bar, the subsequent path of the mass centre and the success of the performance.

\section{CONCLUSION}

Differences between successful and unsuccessful performances were identified from the parameters at release, in particular the horizontal velocity of the mass centre. Less obvious, was the difference in velocity of the mass centre relative to the bar at regrasp. For consistent successful regrasping a radial velocity towards the bar appears to be preferable. The successful gymnast had larger and more consistent release windows, however both gymnasts were equally consistent in the technique used. Although the less successful gymnast had a consistent technique a small change in timing resulting in an earlier closing of the shoulder and hip angles led to a more appropriate flight path for regrasping the bar.

\section{Conflict of Interest Statement}

The authors wish to disclose that they have no financial or personal relationships with any people or organisations that could inappropriately influence this work.

\section{Acknowledgements}

The authors would like to thank the gymnasts and coaches who participated in the study for their time and expertise.

\section{REFERENCES}

Arampatzis, A., Brüggemann, G.-P., 1999. Mechanical energetic processes during the giant swing exercise before dismounts and flight elements on the high bar and the uneven parallel bars. Journal of Biomechanics 32, 811-820.

Begon, M., Wieber, P-B., Yeadon, M.R., 2008. Kinematics estimation of straddled movements on high bar from a limited number of skin markers using a chain model. Journal of Biomechanics 41, 581-586. 
Brüggemann, G-P., Cheetham, P.J., Alp, Y., Arampatzis, D., 1994. Approach to a biomechanical profile of dismounts and release-regrasp skills of the high bar. Journal of Applied Biomechanics 10, 291-312.

Carroll, D.L., 2001. FORTRAN genetic algorithm driver. Downloaded from: http://cuaerospace.com/carroll.ga.html

Cohen, J., 1988. Statistical power analysis for the behavioral sciences (2nd ed.). Hillsdale, NJ: Lawrence Earlbaum Associates.

Cohen, R.G., Sternad, D., 2009. Variability in motor learning : relocating, channeling and reducing noise. Experimental Brain Research 193, 69-83.

Fédération Internationale de Gymnastique, 2013. Code de pointage: Gymnastique artistique masculine [Code of points: Artistic gymnastics for men]. Lausanne.

Forrester, S.E., Yeadon, M.R., King, M.A., Pain, M.T.G., 2011. Comparing different approaches for determining joint torque parameters from isovelocity dynamometer measurements. Journal of Biomechanics 44, 955-961.

Gervais, P., Tally, F., 1993. The beat swing and mechanical descriptors of three horizontal bar release-regrasp skills. Journal of Applied Biomechanics 9, 66-83.

Hiley, M.J., Yeadon, M.R., Buxton, E., 2007. Consistency of performance in the Tkatchev release and re-grasp on high bar. Sports Biomechanics, 6 (2), 119-128.

Hiley, M.J., Yeadon, M.R., 2003a. Optimum technique for generating angular momentum in accelerated backward giant circles prior to a dismount. Journal of Applied Biomechanics 19, 119-130.

Hiley, M.J., Yeadon, M.R., 2003b. The margin for error when releasing the high bar for dismounts. Journal of Biomechanics 36, 313-319.

Hiley, M.J., Yeadon, M.R., 2012a. Achieving consistent performance in a complex whole body movement: the Tkatchev on high bar. Human Movement Science 31, 834-843.

Hiley, M.J., Yeadon, M.R., 2012b. The effect of cost function on optimum technique of the undersomersault on parallel bars. Journal of Applied Biomechanics 28, 10 19.

Hiley, M.J., Yeadon, M.R., 2013. Investigating optimal technique in a noisy environment: application to the upstart on uneven bars. Human Movement Science 32, 181-19.

Hiley, M.J., Yeadon, M.R., 2014. Determining the solution space for a co-ordinated whole body movement in a noisy environment: application to the upstart in gymnastics. Journal of Applied Biomechanics 30, 508-513.

Hiley, M.J., Yeadon, M.R., 2016. Investigating optimal technique in the presence of motor system noise: application to the double layout somersault dismount on high bar. Journal of Sports Sciences 34, 440-449.

Hiley, M.J., Zuevsky, V.V., Yeadon, M.R., 2013. Is skilled technique characterised by high or low variability? - An analysis of high bar giant circles. Human Movement Science 31, 171-180.

Holvoet, P., Lacouture, P., Duboy, J., 2002. Practical use of airborne simulation in a release-regrasp skill on the high bar. Journal of Applied Biomechanics 18, 332 344.

Irwin, G., Exell, T.A., Manning, M.L., Kerwin, D.G., 2016. Functional phases and angular momentum characteristics of Tkatchev and Kovacs. Journal of Sports Sciences, available online.

Kerwin, D.G., Yeadon, M.R., Harwood, M.J., 1993. High bar release in triple somersault dismounts. Journal of Applied Biomechanics 9, 279-286. 
King, M.A., Kong, P.W., Yeadon, M.R. 2009. Determining effective subject-specific strength levels for forward dives using computer simulation of recorded performances. Journal of Biomechanics 42, 2672-2677.

van Beers, R.J., Haggard, P., Wolpert, D.M., 2004. The role of execution noise in movement variability. Journal of Neurophysiology 91, 1050-1063.

van Soest, A.J., Cassius, R.L.J., 2003. The merits of a parallel genetic algorithm in solving hard optimiszation problems. Journal of Biomedical Engineering 125, 141-146.

Wilson, C., Yeadon, M.R., King, M.A., 2007. Considerations that affect optimised simulation in a running jump for height. Journal of Biomechanics 40, 3155-3161.

Wood, G.A., Jennings, L.S., 1979. On the use of spline functions for data smoothing. Journal of Biomechanics 12, 477-479.

Yeadon, M.R., 1990a. The simulation of aerial movement - II. A mathematical inertia model of the human body. Journal of Biomechanics 23, 67-74.

Yeadon, M.R., 1990b. The simulation of aerial movement - III: The determination of the angular momentum of the human body. Journal of Biomechanics $23,75-83$. 